\title{
Acknowledgment of Ad Hoc Reviewers
}

The editors thank the following individuals for their assistance with manuscript reviews during the past year.

Beau Abar

Kristen M. Abraham

Kari Adamsons

Nour Al Ghriwati*

David L. Albright

Melissa A. Alderfer

Elizabeth Allen

Samuel H. Allen

Arthur Aron

Didem Aydogan

Dayoung Bae

Sun-hye Bai*

Benjamin Bailey

Bruce L. Baker

Melissa G. Bakhurst

Christina Balderrama-Durbin

Meeta Banerjee

Miya Barnett*

Robin A. Barry

Suzanne Bartle-Haring

Allen Barton*

Donald H. Baucom

Christopher R. Beam

Amy Beaudry*

Marjorie Beeghly

Martha Ann Bell

Noora Berg

Jeffrey S. Bergmann

Lisa Berlin

Annie Bernier

Sara Berzenski

Katie Black ${ }^{*}$

Alysia Y. Blandon

Adrian Blow

Guy Bodenmann

Daniel Bolt

Paola Bonifacci

Julia V. Borisenko

Genevieve Bouchard

Kaitlin Bountress

Susan Branje

Julia Braungart-Rieker

David Bridgett

Alexis Brieant"

Holly Brophy-Herb

Alice M. Brown

Cheryl Buehler

Sherwood Burns-Nader

Dean Busby

Asuman Buyukcan-Tetik

Natasha Cabrera

Courtney Cadieux*

Sarah B. Campbell

Nicole Campione-Barr

Amy Canevello

Amanda Cannarella

Margaret Canter*
Ryan Carlson

Jason S. Carroll

Julie F. H. Cassé

Julie A. Cederbaum

Jason Chapman

Pajarita Charles

Stephen Chen

Kristene Cheung

Junhan Cho

Sook In Cho

Malissa Clark

Jesse Coe

Catherine Cohan

Inbar Cohen*

Stephan Collishaw

James V. Cordova

AliceAnn Crandall

Stephen Cranney

Alexander Crenshaw*

Julie Crouch

Rick A. Cruz

Carolyn E. Cutrona

Danielle Dallaire

Maayan Davidov

Joanne Davila

Pamela Davis-Kean

Janet A. Deatrick

Andres De Los Reyes

Theodore Dix

Meghan R. Donohue

Brian D. Doss

Kimberly Dossett*

Taylor Dovala*

Joan Durrant

Veroni Eichelsheim

Gregory Elliott

Lindsey Elliott"

Gary W. Evans

Robin S. Everhart

Jay Fagan

Reza Fallahchai

Rachel H. Farr

Allison K. Farrell

Laura J. Finan

Eric Finegood

Adam R. Fisher

Alison Fleming

Eirini Flouri

Aaron Fobian

Alexis R. Foulstone

Steffany J. Fredman

Jayne A. Fulkerson

James L. Furrow

Issac Galatzer-Levy

Sarah Galdiolo
Martin I. Gallegos

Lawrence Ganong

Darren J. Garcia**

Sarah Garcia*

Terese Glatz

Rachel Goldberg*

Susan E. Golombok

Andrea Gonzalez

Jan Greenberg

Katherine Grein*

James Griffith

Andrew Grogan-Kaylor

Joseph Grzywacz

Hanna C. Gustafsson

Daniel Gutierrez

Thao Ha

Kurt Hahlweg

Amber Hammons

Katja Hanke

Amanda Hasselle*

Alan J. Hawkins

Chris L. Heavey

Kristin Heron

Leah C. Hibel

Edward J. Hill

Ben Hinnant

Celine Hinnekens*

Jasara Hogan*

Ashley Holland

Marissa Holst

Rebecca Horne*

Kathryn H. Howell

Alissa Huth-Bocks

Laudan B. Jahromi

Jeremy Jamieson

Lisa Jaremka

Shanuki D. Jayamaha

Corinna Jenkins Tucker

Jennika Jenkins*

Linqin Ji

Matthew D. Johnson

Charlotte Johnston

Blake L. Jones

Joseph W. Jones*

Benjamin R. Karney

Deborah Kashy

Mary Keeling

Amy Kennedy Root

Chandra E. Khalifian

Elizabeth J. Kiel

Ji-Yeon Kim

Jungmeen Kim-Spoon

Jeffrey B. Kingree

Noona Kiuru

Olli Kiviruusu*

Wendy Kliewer
Naomi B. Knoble*

Catherine Kuhns*

Melissa Kull

Patty Kuo

Sally I-Chun Kuo

Brian Lakey

Adam D. LaMotte

Michael R. Langlais

Robert E. Larzelere

Justin Lavner

Daniel J. Laxman

Yunying Le*

Esther Leerkes

Liliana J. Lengua

Ken Leonard

Danli Li

Shaozhuan Li

Jeffrey Liew

Kristin M. Lindahl

Jallu Lindblom

Juhong Liu

Rachel G. Lucas-Thompson

Linda J. Luecken

Erika Lunkenheimer

Shelley M. MacDermid

Wadsworth

Jacqui A. Macdonald

Samantha Miadich*

Katie Maguire Jack

Emily Maher*

Annette Mahoney

Esther Malm*

Anthony Mancini

Jay Mancini

Katja Margelisch

Emma M. Marshall

Sheila Marshall

Meredith Martin

Marisa Matias

Richard E. Mattson

Michael J. McCarthy

Nancy L. McElwain

Meghan M. McGinn

Laura A. Meis

Janet N. Melby

Kristin Mickelson

Anne Milek

Laura E. Miller-Graff

Kristin Moilanen

Amy R. Monn

Ginger Moore

Danyel A. V. Moosmann

Todd Morrison

Maryam Motia*

Liz Munoz-Diaz

Kyle W. Murdock 
Lisa A. Neff

Charles Negy

Jenae M. Neiderhiser

Jackie A. Nelson

Adam Newton*

Tamara Newton

Jerika Norona*

Amy K. Nuttall

Jelena Obradovic

Catherine W. O'Neal

David Oppenheim

Laura M. Padilla-Walker

F. Giorgia Paleari

Justin Parent

Jack S. Peltz

Jemima Petch

Randi Phelps*

Lauren E. Philbrook

Andre Plamondon*

Elizabeth M. Planalp

Christin L. Porter

Julie Premo

Kathleen S. J. Preston

Jacob B. Priest

Sam Putnam

Tara Queen

Marcela Raffaelli

Emily Raiche*
Nairan Ramirez-Esparza

Maryna Raskin

Niyantri Ravindran*

Holly E. Recchia

Bonney Reed-Knight

Chelsea A. Reid

Graham J. Reid

Harry Reis

Heidi R. Riggio

Anne Riley

Jennifer Ripley

Lyndon A. Riviere

Matthew A. Robinson

Kathleen M. Roche

Aubrey J. Rodriguez

Natalie O. Rosen

Wendy Rote

Frances Ruiz-Alfaro

Helena Rutherford

Shiri Sadeh-Sharvit

Catherine D. Santiago

Catalina Sarmiento*

Joni Sasaki

Frederic J. Sautter

Steven L. Sayers

Dave Sbarra

Alice C. Schermerhorn

Karen Schmaling

Thomas J. Schofield

Kyleigh Schraeder*

Erin Shumlich*
Stacey Scott

James P. Selig

Anne Shaffer

Erin S. Sheets

Elizabeth C. Shelleby

Katherine Shelton

Michelle Sherman

Carol K. Sigelman

Jeffry Simpson

Judith G. Smetana

David A. Smith

Tim Smith

Derek Spangler

Maria Spinelli

Mark Stanton

Tyrel J. Starks

Catherine H. Stein

Matthew Stevenson

Beth Stormshak

$\mathrm{Hui} \mathrm{Su}$

Jennifer Suor

Gali Tangir

Fiona Tasker

Zoe E. Taylor

Jennifer Theule

Eric Thibodeau

Theodore Tomeny

Monica Tomlinson*

Martha C. Tompson

Thomas E. Trail

Joseph M. Trombello

Wendy M. Troxel

Kelly M. Tu
Erin C. Tully

Kathryn Turnbull*

Brad Van Eden-Moorefield

Marije Verhage

Lesley Verhofstadt

Marie-Helene Veronneau

Martha Wadsworth

Zhe Wang

Chelsea M. Weaver

Jennifer M. Weaver

Melanie Wheatley*

Lorey A. Wheeler

Mikhila Wildey

Hannah C. Williamson

Brian Willoughby

Steven R. Wilson

Michael Windle

Sharlene Wolchik

Erica Woodin

Laura Wray-Lake

Brian Wymbs

Ni Yan

Kristen Yule*

Maureen Zalewski

Osnat Zamir

Sally Zheng*

Belal Zia*

Bharathi J. Zvara

\footnotetext{
* Denotes a reviewer who coreviewed under the supervision of a primary reviewer.
} 